FOLIA

Amazónica

Revista del Instituto de Investigaciones

de la Amazonía Peruana

\title{
CARACTERIZACIÓN MORFOLÓGICA Y CLAVE DENDROLÓGICA DE CUATRO ESPECIES FORESTALES DEL ARBORETUM DE LA FACULTAD DE CIENCIAS AGRARIAS - UNIVERSIDAD NACIONAL DEL NORDESTE (FCA-UNNE), CORRIENTES, ARGENTINA
}

\author{
Virginia T. BIANCIOTTO ${ }^{1}$, María L. FONTANA ${ }^{1,2}$, Claudia V. LUNA ${ }^{3}$ \\ 1 Universidad Nacional del Nordeste. Facultad de Ciencias Agrarias. Sargento Cabral 2131, W3402BKG \\ Corrientes, Argentina. \\ 2 Instituto Nacional de Tecnología Agropecuaria. Estación Experimental Corrientes. Corrientes, \\ Argentina. \\ 3 Universidad Nacional del Nordeste. Facultad de Ciencias Agrarias. Instituto de Botánica del Nordeste \\ (IBONE) - CONICET. Sargento Cabral 2131, W3402BKG Corrientes, Argentina. Correo electrónico: \\ claudiaverluna@gmail.com
}

\section{RESUMEN}

En los bosques y plantaciones la identificación errónea de especies de árboles en las primeras etapas de su vida puede tener efectos ecológicos y económicos devastadores. Desafortunadamente,enalgunas regiones, lasclaves dendrológicas de especies difíciles de identificar aún no se han desarrollado. Evaluamos las características dendrológicas de cuatro especies de árboles (Delonix regia, Enterolobium contortisiliquum, Jacaranda mimosifolia, Peltophorum dubium) dentro del Arboretum de la Facultad de Ciencias Agrarias - Universidad Nacional del Nordeste (FCA-UNNE), Corrientes, Argentina. Todas las especies comparten características similares (por ejemplo, hojas) durante las etapas de crecimiento vegetativo y juveniles. Se recolectaron, fotografiaron e identificaron muestras botánicas de los árboles por especies comparando muestras con especímenes de herbario del Instituto de Botánica del Nordeste (IBONE), Argentina. Nuestra descripción de cada especie se basó en las características morfológicas y organolépticas del tallo, ramas, hojas, flores, frutos, diámetro a la altura del cuello (DAC), bifurcación y estado de salud. También recopilamos información de la literatura relacionada con la distribución geográfica en Argentina, el estado de conservación y otra información ecológica relevante. Estas nuevas descripciones de especies y claves dendrológicas pueden facilitar la identificación de campo rápida y precisa para especies de árboles ecológica y 
económicamente importantes en etapas tempranas de vida cuando parecen ser morfológicamente similares.

PALABRAS CLAVE: Delonix regia, Enterolobium contortisiliquum, Jacaranda mimosifolia, Peltophorum dubium, aspectos dendrológicos, especies arbóreas.

\title{
MORPHOLOGICAL CHARACTERIZATION AND DENDROLOGICAL KEY OF FOUR FOREST SPECIES OF THE FACULTAD DE CIENCIAS AGRARIAS - UNIVERSIDAD NACIONAL DEL NORDESTE (FCA-UNNE) ARBORETUM
}

\begin{abstract}
In managed forests and plantations, misidentification of tree species in early life stages may have devastating ecological and economic impacts. Unfortunately, in some regions, dendrological keys of difficult to identify species have yet to be developed. We assessed dendrological characteristics of four tree species (Jacaranda mimosifolia, Peltophorum dubium, Enterolobium contortisiliquum and Delonix regia) within the Arboretum at the Facultad de Ciencias Agrarias - Universidad Nacional del Nordeste (FCA-UNNE), Corrientes, Argentina. All species share similar characteristics (e.g., leaves) during seedling and vegetative growth stages. Botanical samples of the trees were collected, photographed, and identified to species by comparing samples to herbarium specimens from the Instituto de Botánica del Nordeste (IBONE), Argentina. Our description of each species was based on morphological and organoleptic characteristics of the stem, branches, leaves, flowers, fruits, Diameter at Neck Height (DNH), bifurcation and health status. We also compiled information from the literature related to geographical distribution in Argentina, conservation status, and other relevant ecological information. These new species descriptions and dendrological keys may facilitate rapid and accurate field identification for ecologically and economically important tree species in early life stages when they appear to be morphologically similar.
\end{abstract}

KEYWORDS: Delonix regia, Enterolobium contortisiliquum, Jacaranda mimosifolia, Peltophorum dubium, dendrological aspects, tree species. 


\section{INTRODUCCIÓN}

La dendrología es una disciplina sumamente importante para las ciencias forestales, aunque se encuentra poco desarrollada en los trópicos de América.

Ríos (1990) menciona que para llevar a cabo la identificación de las especies forestales a nivel dendrológico, debe estudiarse las características vegetativas del individuo (posición de la hoja, tipo de hoja entre otras) ya a nivel taxonómico se resaltan los caracteres reproductivos del individuo (flor y fruto).

La menor variabilidad fenotípica en el tiempo y el espacio de los órganos reproductivos (flores $\mathrm{y}$ frutos) frente a otras partes vegetativas, es un factor que favorece que los estudios taxonómicos destinados a la identificación y la clasificación se centren en la morfología de las flores, siendo esta la base para la clasificación de todas las Angiospermas. Este aspecto, unido a los complejos hábitos de floración y fructificación, ha generado que la bibliografía dendrológica existente sea escasa y fragmentaria, abundando las descripciones florísticas que usan lenguaje poco usado por los forestales y las personas interesadas (Kroll et al., 1992).

Los estudios de caracterización dendrológica de las principales especies forestales resultan ser de vital importancia para la formulación de planes de manejo forestal sostenible y para el proceso de certificación forestal, ya que nos permite realizar de manera adecuada el aprovechamiento y el manejo sostenible del recurso forestal (Dueñas Linares \& Nieto Ramos, 2010).

Los especialistas en dendrología saben que hay una serie de características fundamentales, como por ejemplo las hojas (dimensión, pubescencia y otros), que dependen delaubicación fitogeográfica del individuo. El tamaño también puede variar según la edad. La forma biológica y el hábito de un árbol son características de suma importancia taxonómica para el dendrólogo, al igual que la anatomía comparada de sus maderas (Comellas García-Llera, 2011).

En este sentido, la dendrología es una herramienta de gran utilidad, ya que mediante las características simples de los órganos vegetativos conjugados realiza el reconocimiento "in situ" e inmediato de los árboles (Giménez \& Moglia, 2003). Algunas características, como por ejemplo las modificaciones de la base del fuste, el tipo de fuste, las variantes de corteza externa, la corteza interna, las secreciones y los olores característicos, son de fácil observación y, usualmente, no son tenidas en cuenta por los botánicos en la identificación de los árboles (Peña, 2013).

La identificación basada en las características dendrológicas constituye una alternativa válida hoy en día para superar dificultades y limitaciones existentes en los procesos de identificación de especies forestales, como por ejemplo la falta de equipos de laboratorio, la carencia de material bibliográfico para las consultas, etc. (Baluarte Vásquez, 1993).

Para poder elaborar de forma correcta las claves se debe tener en cuenta algunas reglas (Marzocca, 1985; Fontúrbel et al., 2007), en las que se incluye atender los estadios juveniles y adulto; ya que pueden presentar diferencias sobre todo en los caracteres vegetativos, como ocurre en las especies objeto de estudio.

Existen antecedentes de caracterización dendrológica para la especie Delonix regia (Bojer ex Hook.) Raf. (Fabaceae), que por ser una especie cosmopolita y de interés ornamental ha demostradomayorinterés en estecampo (Quesada Monge \& Fernández Vega, 2005; Rodríguez Aranguren \& Gámez Álvarez, 2010; Aguirre, 2012; SINAC, 2014). Para las especies nativas propuestas en este trabajo, Jacaranda mimosifolia D. Don (Bignoniaceae), Enterolobium contortisiliquum (Vell.) Morong. y Peltophorum dubium (Spreng.) Taub. (Fabaceae), los antecedentes al respecto 
son escasos (Killeen et al., 1993; Mostacedo et al., 2003; Amurrio Mamani, 2009; Yajure \& Gámez, 2011).

La región amazónica presenta una gran diversidad de plantas nativas, muchas de las cuales no cuentan con estudios científicos específicos, siendo utilizadas, no obstante, con sabiduría por los aborígenes de la zona (Yépez, 2011; Tapia \& Armas, 2014). En la Amazonía, la familia Bignoniaceae se encuentra representada por el género Jacaranda (Zarate Gómez et al., 2015), siendo usada de manera ancestral por las comunidades amazónicas (Tapia \& Armas, 2014). Existe registro de una clave dicotómica para la identificación de dos especies en particular de este género, realizado por Zarate Gómez et al. (2015), relevadas en los bosques sobre arena blanca de la Reserva Nacional Allpahuayo Mishana (Perú).

Por otra parte, existen experiencias en la cuenca amazónica (Brasil, Bolivia y Perú), que registran al género Peltophorum como una de las especies de regeneración tolerada en los pastizales de la Amazonía y a Enterolobium como uno de los géneros comerciales de maderas más usadas de la región (Egg, 1994).

El objetivo del presente trabajo consistió en la caracterización morfológica y la confección de claves dendrológicas para estado vegetativo y reproductivo de cuatro especies forestales del Arboretum de la Facultad de Ciencias Agrarias, Universidad Nacional del Nordeste (FCA-UNNE) en Corrientes, Argentina.

\section{MATERIAL Y MÉTODOS}

\section{ÁREA DE ESTUDIO}

El Arboretum de la Facultad de Ciencias Agrarias, Universidad Nacional del Nordeste (FCA-UNNE) abarca alrededor de 2 ha y se encuentra ubicado en el Campus Deodoro Roca de la Universidad Nacional del Nordeste (UNNE), localizado en la ciudad de Corrientes, Argentina (latitud

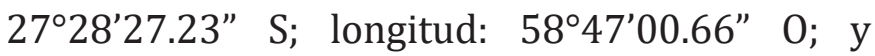
altitud: $50 \mathrm{msnm}$ ). Se encuentra ubicado a $8 \mathrm{~km}$ del microcentro, en el cuadrante noreste de la trama urbana y tiene accesos por la Avenida Libertad y por la Ruta Nacional $\mathrm{N}^{\circ} 12$. Por esta última, a la altura del $\mathrm{km}$ 1,031, se accede directamente al sector del Campo Didáctico Experimental Agrícola (CDEA) de la Facultad de Ciencias Agrarias (FCA), Universidad Nacional del Nordeste (UNNE), donde se ejecuta el proyecto en cuestión (Figura 1) (Sugita et al., 2018).

\section{CARACTERIZACIÓN CLIMÁTICA Y TOPOGRÁFICA DEL ÁREA DE ESTUDIO}

Se presentan precipitaciones promedio anuales de $1,300 \mathrm{~mm}$, y temperaturas medias anuales de $21,6^{\circ} \mathrm{C}$. El período libre de heladas es de 340 a 360 días por año y su frecuencia de ocurrencia es de 0,5. Tomando la clasificación de Köppen modificada, el clima en la región se clasifica como mesotermal húmedo, Cf w’a (h) (Murphy, 2008). El departamento Capital de la provincia de Corrientes se encuentra en la línea de isoterma de $22^{\circ} \mathrm{C}$ y entre las isohietas de 1,300 y $1,400 \mathrm{~mm}$ (Sugita et al., 2018).

En cuanto a las condiciones del terreno, se determinó que existe una pendiente del 1,92\% en el área del arboreto, teniendo en cuenta el punto más alto y bajo del terreno, en dirección suroestenoreste. El área de estudio presentó las mismas características descriptas por Escobar et al. (1996) para toda la provincia de Corrientes; son suelos que se encuentran sometidos a una continua edafización debida a las lluvias abundantes y a las altas temperaturas, que induce la formación de suelos ácidos, reflejados en sus horizontes eluviales y en los subyacentes. Los estudios del perfil del suelo del sitio se encuentran en la serie de suelo Paso Patria.

La profundidad efectiva se extiende hasta los $70 \mathrm{~cm}$. El drenaje es imperfecto, con escurrimiento 
Figura 1. Ubicación del Arboretum de la Facultad de Ciencias Agrarias, Universidad Nacional del Nordeste. La flecha indica la ubicación del predio en estudio. Fuente: Google Maps 2020.

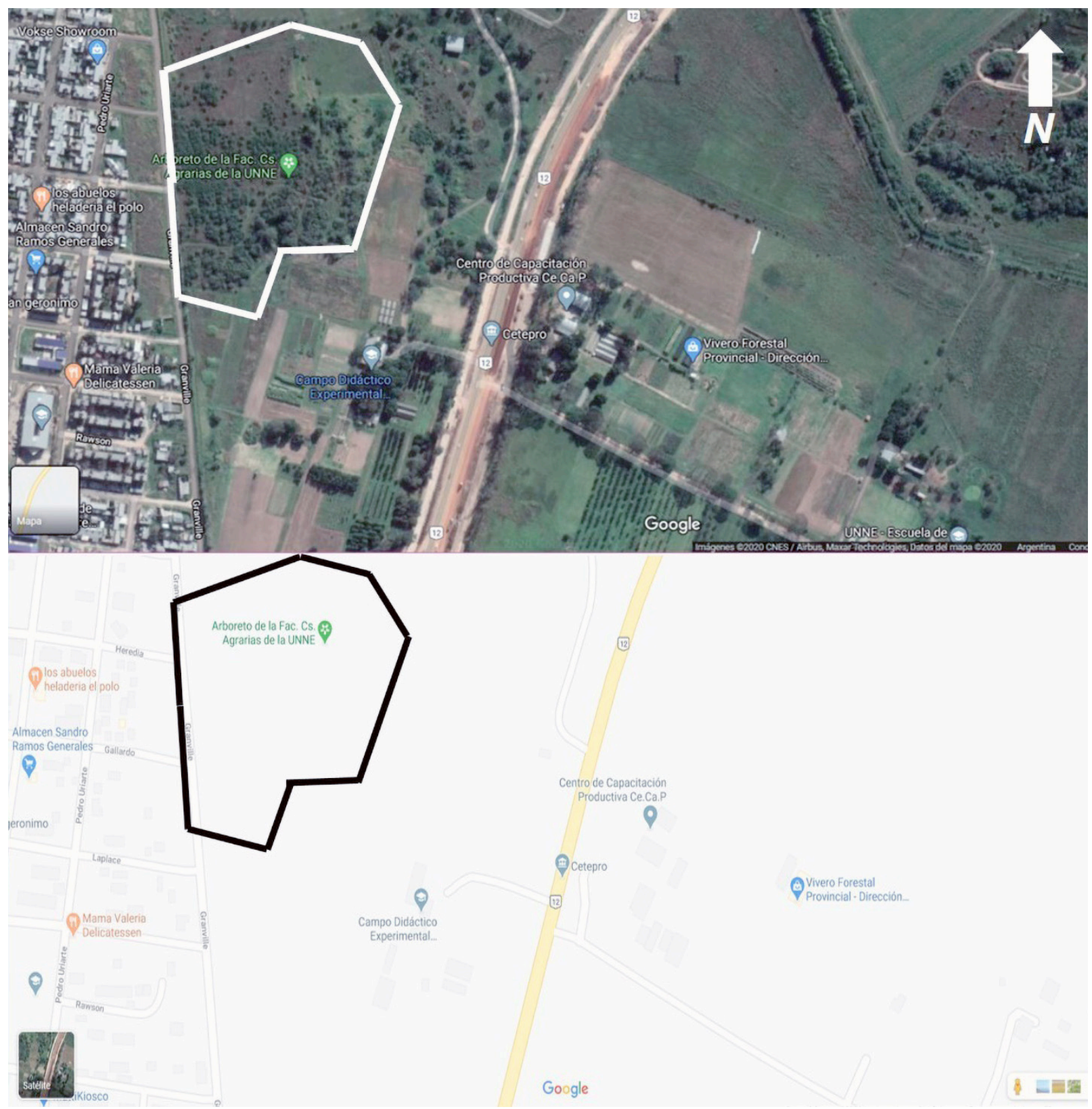

muy lento y permeabilidad lenta, inundables a poco inundables, generalmente en época de grandes crecientes (Escobar et al., 1996). Estas características otorgan al suelo del sitio una capacidad de uso VI, es decir, que tiene graves limitaciones que lo hace no apto para el desarrollo de cultivos (Sugita et al., 2018).

\section{CARACTERIZACIÓN DENDROLÓGICA}

Para la caracterización de los árboles se utilizó una ficha dendrológica previamente diseñada (Dueñas Linares \& Nieto Ramos, 2010). Anotándose las siguientes informaciones: DAC (diámetro a la altura del cuello), altura total, habito, ramificación, fuste, raíz, corteza externa 
(color); corteza interna (color); hojas (tipo de hojas, filotaxis, pubescencia del raquis, simetría y venación foliolos/foliólulos); inflorescencia (tipo y color); tipo de fruto y semillas, y estado sanitario. Además, se complementaron para cada especie información sobre fenología (época de floración y fructificación), distribución geográfica en Argentina, estado de conservación e información ecológica (altitud, hábitat, especies asociadas, usos).

REGISTRO DE FOTOGRAFÍAS DE LOS ÁRBOLES Se utilizó una cámara fotográfica digital General Electric de 12.2 Megapíxeles y 5X de Zoom, las imágenes fueron codificadas de acuerdo a los caracteres de cada árbol. Las imágenes registradas fueron del árbol completo, copa de árbol, ramificaciones, fuste, corteza externa, hojas (disposición, borde, ápice, base, presencia de estipulas, peciolo, etc.) (Dueñas Linares \& Nieto Ramos, 2010)

\section{IDENTIFICACIÓNDEESPECÍMENESVEGETALES}

Se identificaron los especímenes a nivel de especie. Se realizaron comparaciones del material colectado en el Herbario CTES del IBONE (Instituto de Botánica del Nordeste). Además, se utilizaron claves dicotómicas, literatura especializada, guías, manuales, floras, etc. (Dueñas Linares \& Nieto Ramos, 2010).

\section{ANÁLISIS DE LOS DATOS DE CAMPO}

Para el análisis de los datos de campo, se revisaron los formularios dendrológicos, libretas de campo y fotografías. Se diseñó un formato en una hoja de cálculo Excel en base a los datos.

\section{CLAVE DENDROLÓGICA}

En la elaboración de la clave se tomó en cuenta las características de los órganos vegetativos y reproductivos y elaborada de manera independiente, permitiendo que cualquier persona con material vegetativo $\mathrm{y} / \mathrm{o}$ reproductivo en mano, pueda seguirla hasta llegar a diferenciar e identificar el espécimen a nivel de especie (Cálix Pizatti, 1970; Sauñe Ferrel, 2013).

\section{RESULTADOS Y DISCUSIÓN}

\section{DESCRIPCIÓN DENDROLÓGICA DE LOS INDIVIDUOS OBSERVADOS}

Los datos relevados a partir de los individuos observados, en buen estado general (hojas enteras, completas y maduras), fértiles (con flores, frutos) y con un buen estado sanitario, hicieron posible un registro de caracteres de importancia para su identificación (Tabla 1 y 2), además de una apreciación de los caracteres morfológicos en estado vegetativo y reproductivo (Tabla 3 y 4 respectivamente), para luego ser complementado mediante una revisión bibliográfica, con las características generales de las especies en estudio.

\section{COMPARACIÓN ENTRE ESPECIES EN ESTADIO VEGETATIVO}

En estadios tempranos, si bien el primer y segundo par de hojas o eófilos, son particulares para cada especie también son elementos que dificultan su identificación; ya que en especies de un mismo género $y$ aún en diferentes géneros de la misma familia pueden presentar similitudes, por ejemplo, la presencia de estípulas en la mayoría de las especies de Fabaceae, en Enterolobium contortisiliquum y Peltophorum dubium, se presenta este caso (Duke, 1969; Parra, 1983). Otra similitud entre E. contortisiliquum y $P$. dubium son las superficies de las cicatrices foliares que pueden ser planas (la mayoría de los casos); pero en estas denominamos de ménsula (Gartland, 2008a).

Un carácter semejante que presentan P. dubium y Delonyx regia es la presencia de estípulas 
compuestas (lámina partida) que se muestran como un par de estípulas libres pinadas (Gartland, 2008a).

Enterolobium contortisiliquum presenta como característica propia, estipelas lineares y pequeñas, que son análogas a las estípulas y se encuentran en la base de los folíolos y pinas en las hojas compuestas (Gartland, 2008b). Además que comienza su estadio temprano con hojas pinnadas y siguen con hojas bipinnadas (Piedrahita, 1987; Duke, 1969; Duke \& Polhill, 1981). Otra particularidad que presenta esta especie solo en las hojas juveniles es la presencia de lenticelas conspicuas que se pierden en su adultez (Gartland, 2008a).

En cuanto a los folíolos asimétricos de varias especies de Fabaceae, presentan esta característica debido a que la vena central está desplazada hacia uno de los bordes y entonces se denomina submarginal; este carácter se puede apreciar como distintivo en E. contortisiliquum. Esta morfología puede ser confundida si se analiza una hoja aislada de Jacaranda mimosifolia, pero la misma se debe a que sus folíolos son oblongos, mucronados y el terminal es largamente rombo elíptico, agudo y acuminado (Gartland, 2008b).

En Peltophorum dubium, si bien las hojas bipinadas caracterizan a la mayoría de las Fabaceae, esta especie presenta hoja compuesta paribipinada; otra característica como especie con hojas estipuladas es la presencia de cicatrices estipulares, cuyas formas son puntiformes (Gartland, 2008a) y además pecíolo y raquis pulverulentos (Kostlin, 2017); teniendo en cuenta estas particularidades se diferencia de E. contortisiliquum porque en esta última son lineares.

Jacaranda mimosifolia presenta las superficies de cicatrices foliares cóncavas (Gartland, 2008a). El órgano foliar muestra una gran variación; no solo en la forma externa y sino en su estructura interna, además de su función. La forma de la hoja está determinada genéticamente $y$, asimismo se puede modificar en adaptación a determinadas condiciones ambientales (Nultsch, 2000). Según Damião Filho \& Môro (2005) la hoja, presenta engrosamientos situados en la base o el ápice del pecíolo y en los peciolulos de sus hojas; denominados pulvinos o pulvínulos respectivamente, según corresponda (Silva Costa et al., 2011).

Delonix regia presenta raquis angulares a levemente acanalados; pilosos en las partes más jóvenes; raquis secundarios terete o con sección transversal que es circular, como un círculo distorsionado, con una sola superficie que lo envuelve; pilosos de tricomas simples poco visibles (Rodríguez Aranguren \& Gámez Álvarez, 2010). 
Tabla 1. Descripción dendrológica de los individuos juveniles observados en el Arboretum de la Facultad de Ciencias Agrarias - Universidad Nacional del Nordeste (FCA-UNNE) Corrientes, Argentina. DAC (diámetro a la altura de cuello).

\begin{tabular}{lcccc}
\hline \multicolumn{1}{c}{ Especies } & Delonix regia & $\begin{array}{c}\text { Enterolobium } \\
\text { contortisiliquum }\end{array}$ & $\begin{array}{c}\text { Jacaranda } \\
\text { Mimosifolia }\end{array}$ & $\begin{array}{c}\text { Peltophorum } \\
\text { Dubium }\end{array}$ \\
\hline $\begin{array}{l}\text { No de plantas } \\
\text { observadas in situ }\end{array}$ & 2 & 4 & 2 & 3 \\
DAC $(\mathrm{cm})$ & $3,40 \pm 0,51$ & $2,74 \pm 0,52$ & $2,94 \pm 1,13$ & $3,07 \pm 0,75$ \\
Altura $(\mathrm{cm})$ & $91,25 \pm 12,23$ & $86 \pm 13,44$ & $107,92 \pm 11,58$ & $103,5 \pm 14,54$ \\
Bifurcación & Sí & No & Sí & No \\
Estadio & Juvenil & Juvenil & Juvenil & Juvenil \\
Estado sanitario & Bueno & Bueno & Bueno & Bueno \\
\hline
\end{tabular}

Tabla 2: Periodos de floración y fructificación de las especies registradas en el Arboretum de la Facultad de Ciencias Agrarias - Universidad Nacional del Nordeste (FCA-UNNE) reportados para Argentina.

\begin{tabular}{|c|c|c|c|}
\hline Especies & Periodo de floración & Periodo de fructificación & Referencias \\
\hline Delonix regia & $\begin{array}{l}\text { Finales de primavera } \\
\text { y verano (noviembre a } \\
\text { febrero) }\end{array}$ & Febrero a octubre & $\begin{array}{l}\text { Grau \& Kortsarz 2012; EOL, } \\
2020 .\end{array}$ \\
\hline $\begin{array}{l}\text { Enterolobium } \\
\text { contortisiliquum }\end{array}$ & $\begin{array}{l}\text { Finales de primavera, } \\
\text { inicio verano (noviembre y } \\
\text { diciembre) }\end{array}$ & $\begin{array}{l}\text { Verano a principio de otoño } \\
\text { (diciembre a marzo) }\end{array}$ & $\begin{array}{l}\text { Salazar \& Soihet, 2001; } \\
\text { Cabrera et al., 2013; Ventoso } \\
\text { \& Mongiardino, } 2014\end{array}$ \\
\hline $\begin{array}{l}\text { Jacaranda } \\
\text { mimosifolia }\end{array}$ & $\begin{array}{l}\text { Finales de verano o } \\
\text { principios de otoño/ } \\
\text { primavera (octubre) }\end{array}$ & $\begin{array}{l}\text { Finales de otoño y } \\
\text { permanecen todo el año }\end{array}$ & $\begin{array}{l}\text { Lema Aucacama, 2013; } \\
\text { Asesor, } 2019 .\end{array}$ \\
\hline $\begin{array}{l}\text { Peltophorum } \\
\text { dubium }\end{array}$ & Verano y otoño & Otoño e invierno & $\begin{array}{l}\text { Cabrera et al., 2013; Ventoso } \\
\text { \& Mongiardino, } 2014\end{array}$ \\
\hline
\end{tabular}


Tabla 3: Caracteres morfológicos en estado vegetativo de las especies observadas en el Arboretum de la Facultad de Ciencias Agrarias - Universidad Nacional del Nordeste (FCA-UNNE) Corrientes, Argentina.

\begin{tabular}{|c|c|c|c|c|}
\hline Especies & $\begin{array}{l}\text { Delonix } \\
\text { regia }\end{array}$ & $\begin{array}{l}\text { Enterolobium } \\
\text { contortisiliquum }\end{array}$ & $\begin{array}{l}\text { Jacaranda } \\
\text { mimosifolia }\end{array}$ & $\begin{array}{l}\text { Peltophorum } \\
\text { dubium }\end{array}$ \\
\hline \multicolumn{5}{|l|}{ Tipo de hojas } \\
\hline \multicolumn{5}{|l|}{ Filotaxis } \\
\hline alternas & $x$ & $x$ & - & $x$ \\
\hline opuestas & - & - & $x$ & - \\
\hline \multicolumn{5}{|l|}{ Venación de foliolos/ } \\
\hline $\begin{array}{l}\text { céntrica } \\
\text { excéntrica }\end{array}$ & - & $x$ & - & - \\
\hline \multicolumn{5}{|l|}{$\begin{array}{l}\text { Simetría de foliolos/ } \\
\text { foliólulos }\end{array}$} \\
\hline simétricos & $x$ & $x$ & $x$ & - \\
\hline asimétricos & - & - & - & $x$ \\
\hline \multicolumn{5}{|l|}{ Pubescencia del raquis } \\
\hline glabro & $x$ & $x$ & $\mathrm{x}$ & - \\
\hline piloso & - & - & - & $x$ \\
\hline \multicolumn{5}{|l|}{ Corteza externa } \\
\hline pardo & $x$ & $x$ & - & - \\
\hline grisácea & - & $x$ & $x$ & $x$ \\
\hline \multicolumn{5}{|l|}{ Corteza interna } \\
\hline castaño claro & $x$ & - & - & - \\
\hline grisácea & - & - & - & $\mathrm{x}$ \\
\hline amarillento & - & - & $x$ & - \\
\hline crema & - & $x$ & - & - \\
\hline \multicolumn{5}{|l|}{ Raíz } \\
\hline pivotante, profunda & - & $x$ & - & $x$ \\
\hline fasciculada & - & - & $x$ & - \\
\hline superficial, gruesa & $x$ & - & - & - \\
\hline
\end{tabular}


Tabla 4: Caracteres morfológicos en estado reproductivo de las especies reportadas en el Arboretum de la Facultad de Ciencias Agrarias - Universidad Nacional del Nordeste (FCA-UNNE), Corrientes, Argentina.

\begin{tabular}{|c|c|c|c|c|}
\hline Especies & $\begin{array}{l}\text { Delonix } \\
\text { regia }\end{array}$ & $\begin{array}{l}\text { Enterolobium } \\
\text { contortisiliquum }\end{array}$ & $\begin{array}{l}\text { Jacaranda } \\
\text { mimosifolia }\end{array}$ & $\begin{array}{c}\text { Peltophorum } \\
\text { dubium }\end{array}$ \\
\hline \multicolumn{5}{|c|}{ Inflorescencia } \\
\hline simple & $x$ & $x$ & - & - \\
\hline compuesta & - & - & $x$ & $x$ \\
\hline \multicolumn{5}{|c|}{ Tipo de inflorescencia } \\
\hline racimo & $x-$ & - & - & - \\
\hline capítulo & & $x$ & - & - \\
\hline panícula & & & $x$ & $x$ \\
\hline \multicolumn{5}{|c|}{ Posición de inflorescencia } \\
\hline terminal & $x$ & $x$ & - & $x$ \\
\hline axilar & $x$ & - & $x$ & - \\
\hline \multicolumn{5}{|c|}{ Color de flores } \\
\hline amarillas & - & - & - & $x$ \\
\hline blancas & - & $x$ & - & - \\
\hline azules & - & - & $x$ & - \\
\hline rojas & $x$ & - & - & - \\
\hline \multicolumn{5}{|c|}{ Tipo de frutos } \\
\hline vaina & $x$ & $x$ & - & - \\
\hline cápsula & - & - & $x$ & - \\
\hline sámara & - & - & - & $x$ \\
\hline \multicolumn{5}{|l|}{ Dehiscencia } \\
\hline sí & $x$ & - & $x$ & - \\
\hline no & - & $x$ & - & $x$ \\
\hline \multicolumn{5}{|l|}{ Semillas } \\
\hline con alas & - & - & $x$ & - \\
\hline sin alas & $x$ & $x$ & - & $x$ \\
\hline
\end{tabular}




\section{CARACTERÍSTICAS DISTINTIVAS DE LAS ESPECIES}

\section{BIGNONIACEAE}

Jacaranda mimosifolia D. Don

- Etimología: Jacarandá: “Jacarandá” en guaraní significa "madera dura" y "mimosifolia" del latín, significa de hojas parecidas a las de una mimosa.

- Nombre común: jacarandá, tarco (Argentina); Avañe'ẽ, jacarandá, karoba, ka'i jepopeté, gejnuá, kekeraundaboom, acpehec, sivavayuc, akpahek (Paraguay); Palisandro del Brasil, flanboyant azul, jakara'nda (Brasil); Gualanday (Colombia)

- Origen: autóctono.

- Distribución geográfica: esta especie es originaria de Brasil y el norte de Argentina: Jujuy, Salta, Tucumán, Catamarca. Nivel inferior de la Selva y en zona de transición con el Parque Chaqueño. Bolivia. Brasil. Paraguay. Se cultiva en las zonas tropicales y subtropicales del mundo, debido a su belleza ornamental (Juárez de Varela, 2012; APN-SIB, 2016).

- Estado de conservación: vulnerable según UICN (Unión Internacional para la Conservación de la Naturaleza) y Lista Roja de la UICN (Prado, 1998). Debido a que su ecosistema natural está siendo rápidamente transformado por la agricultura (UICN, 2017).

- Material consultado en herbario: ARGENTINA. CORRIENTES: Corrientes, Capital. 08/ IX/2008, Schinini, A. 36967. Herbario CTES.

- Información ecológica: la especie crece a bajas elevaciones, en climas húmedos. Deja caer sus hojas durante la estación seca, pero las repone a inicios de la estación lluviosa. Por su atractiva floración púrpura, también es usado como ornamental (Asociación Española de Arboricultura, 2014).

\section{FABACEAE}

Delonix regia (Bojer ex Hook.) Raf.

- Etimología: Delonix, del griego delos = evidente, notable y onus = uña, en referencia a los pétalos notablemente unguiculados; regia, del latín regium-a-um = real, por su grandiosidad cuando está en flor.

- Nombre común: tabachín (México), flamboyán (Guatemala), acacia roja (Colombia y Honduras), árbol de fuego (El Salvador), malinche (Costa Rica) y chivato (Argentina y Paraguay).

- Origen: es originaria de la selva seca caducifolia de Madagascar, en donde está en peligro de extinción. Es uno de los árboles ornamentales más extensamente cultivados en regiones tropicales y subtropicales de todo el mundo y el que se ha naturalizado localmente (AcostaHernández, 2013).

- Distribución geográfica: es endémica de la Isla de Madagascar, pero se encuentra ampliamente distribuida en todo el mundo. También se cultiva en la mayoría de las zonas de Madagascar y en gran parte de los trópicos como ornamental. Se la encuentra desde el nivel del mar hasta los 400 metros, en ocasiones en alturas cercanas a los $750 \mathrm{~m}$ (Rivers, 2014).

- Estado de conservación: preocupación menor (LC), UICN (Rivers, 2014).

- Material consultado en herbario: ARGENTINA. CORRIENTES: Dep. Ledesma, Jujuy. 11/ XI/1992, Kiesling, R. 8154. Herbario CTES.

- Información ecológica: especie de madera débil y a menudo los vientos fuertes rompen los árboles. Después de la caída de las hojas, los árboles se tornan menos atractivos con sus vainas conspicuas colgando de las ramas desnudas, mostrando los túneles y nidos prominentes de las termitas que a menudo atacan esta especie (Elbert et al., 1977). Otra característica indeseable es el sistema radical que a menudo rompe las aceras y paredes. 
Los frutos a veces se usan como instrumentos musicales (Hoyos, 1979).

Enterolobium contortisiliquum (Vell.) Morong.

- Etimología: Enterolobium, del griego entero = intestino y lobion = lóbulo, aludiendo a la forma de sus frutos; contortisiliquum, del latín contortus-a-um $=$ retorcido $\mathrm{y}$ siliqua $=$ vaina, un tipo de fruto capsular seco, aludiendo a la forma de su fruto retorcido.

- Nombre común: araribá, cambanambí, chimbó, tamboi, timbauba, ximbiuva, ximbó, pau sabao (Brasil); toco, timboy (Bolivia); timbó colorado (Paraguay); oreja de negro, pacará (Argentina); timbó (Uruguay).

- Origen: autóctono.

- Distribución geográfica: en Argentina tiene una amplia distribución en la provincia de las Yungas, provincia Paranaense y Distrito chaco Oriental. Chaco, Corrientes, Entre Ríos, Formosa, Jujuy, Misiones, Salta, Santa Fe, Tucumán (APN-SIB, 2016).

- Estado de conservación: aún no ha sido evaluado por la Lista Roja de la UICN, pero se encuentra registrado en The Catalogue of Life (Roskov et al., 2016).

- Material consultado en herbario: ARGENTINA. CORRIENTES: Corrientes, Capital. 01/XI/1975, Maidana, J. 102. Herbario CTES.

- Información ecológica: especie heliófila, poco exigente en cuanto a las características del suelo; de crecimiento rápido (Lozano et al., 2016). Cuando crece fuera de la selva o en regiones próximas a su límite austral de dispersión (Delta del Paraná, isla Martín García) disminuye su estatura y especialmente la longitud del fuste (APN-SIB, 2016).

\section{Peltophorum dubium (Spreng.) Taub.}

- Etimología: Peltophorum, del griego pelte = escudo y phoros = producir, portar, en alusión a los estigmas peltados de la flor; dubium, del latín dubius-a-um = incierto, dudoso, en alusión a su posición taxonómica.

- Nombre común: "ibirá-pitá", es de origen guaraní y significa "ibirá": palo, árbol y "pitá": colorado, aludiendo al color de su madera. Camurim, cana-fístula (Brasil), árbol de Artigas, ibirapitá (Uruguay), yvyrá-pytá, ibirá puitá guazú, o ibirapitá, Yvyra Pytã (Argentina y Paraguay).

- Origen: Autóctono.

- Distribución geográfica: en Argentina esta especie crece naturalmente en las selvas altas de Corrientes, Misiones, Chaco, Formosa y norte de Santa Fe, siendo cultivada además en parques y avenidas de la región templadocálida del país (APN-SIB, 2016).

- Estado de conservación: este taxón aún no ha sido evaluado por la Lista Roja de la UICN y tampoco está incluido en the Catalogue of Life (UICN, 2016).

- Material consultado en herbario: ARGENTINA. CORRIENTES: Corrientes, Capital. 23/ II/1990, Krapovickas, A. 43597. Herbario CTES.

- Información ecológica: especie con crecimiento rápido que prefiere exposición soleada y temperaturas suaves, vegeta bien en varias clases de suelos, excepto los pedregosos o excesivamente húmedos, tolerando bastante bien la sequía, aunque crece mejor en suelos bien drenados y medianamente fértiles (Carvalho, 1994). Es muy utilizada como fijador de médanos, para cortinas rompevientos, en setos vivos altos o para abrigo del ganado. Acepta la poda frecuente (López et al., 1987). Forma parte del listado de especies declaradas Monumentos Naturales de la Provincia de Misiones, lo que implica la prohibición de su apeo, puesto que es una de las especies que se encuentran en una situación de peligro, en riesgo de desaparición (Poulsen et al., 1998). 
FOLIA

Figura 2. Detalle de caracteres dendrológicos vegetativos de Peltophorum dubium: A-hoja; B-foliolos; C-raquis y D-corteza.

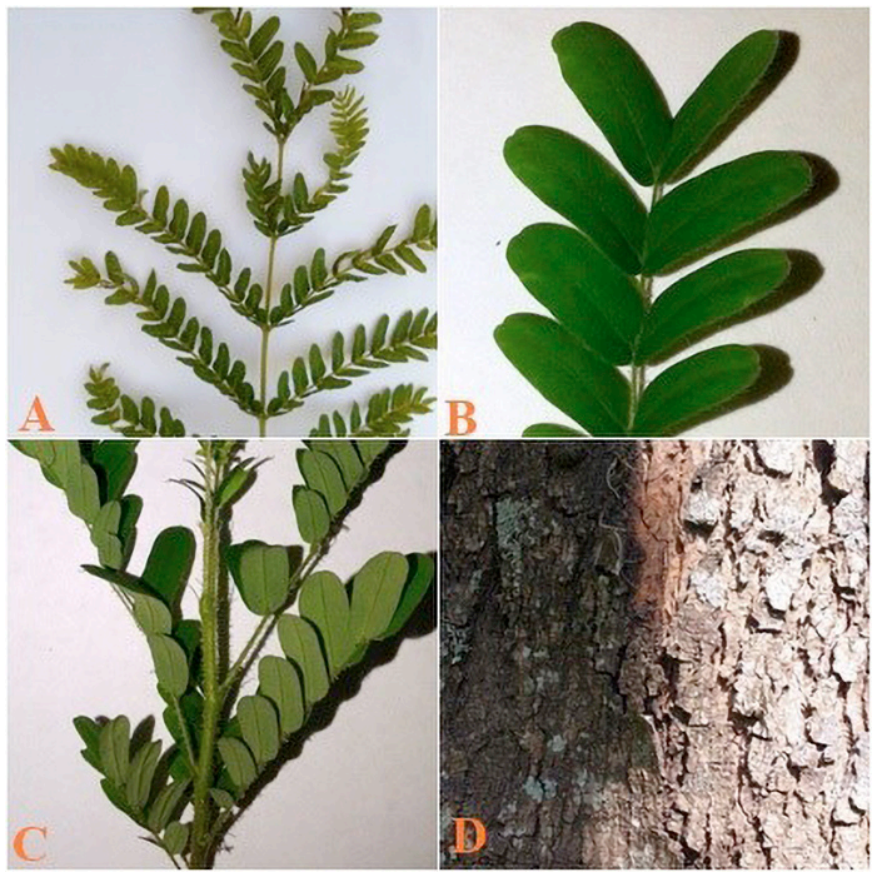

Figura 4. Detalle de caracteres dendrológicos vegetativos de Enterolobium contortisiliquum: A-hoja; B-foliolos; C-raquis y D-corteza.

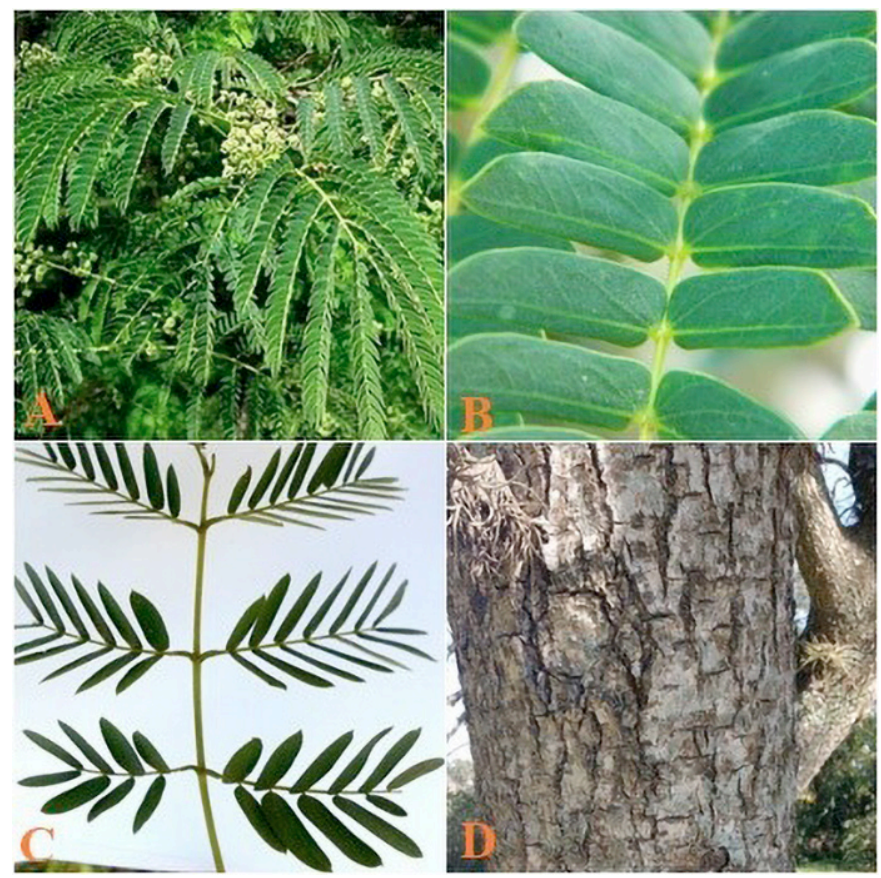

Figura 3. Detalle de caracteres dendrológicos vegetativos de Delonix regia: A-hoja; B-foliolos; C-raquis y D-corteza.

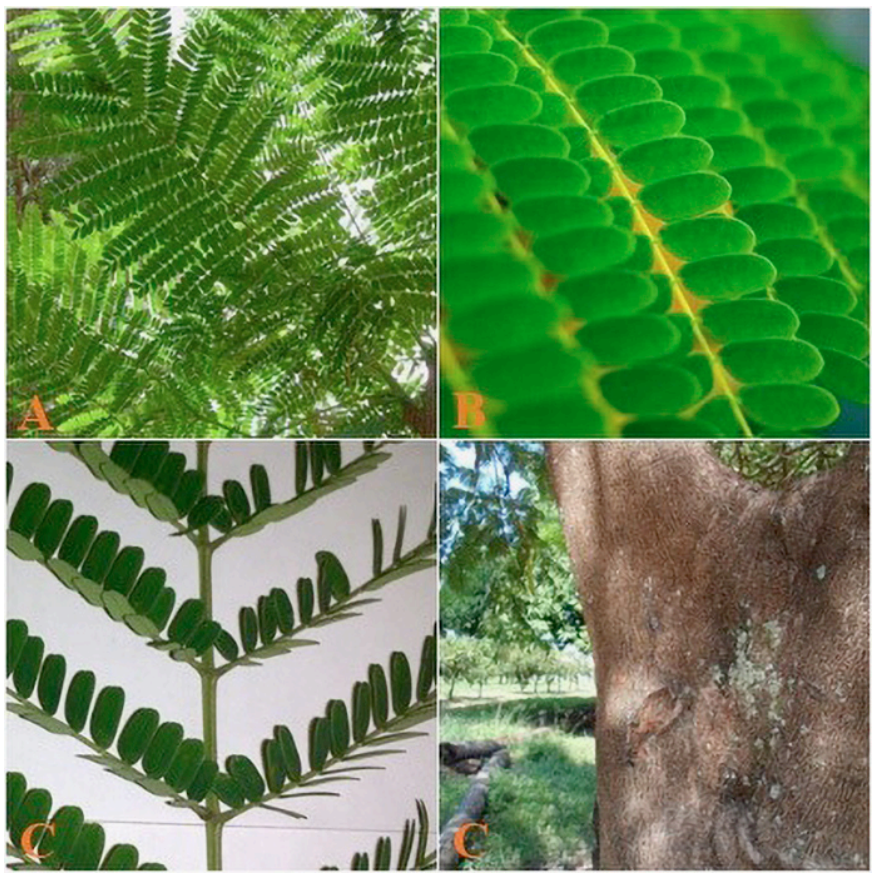

Figura 5. Detalle de caracteres dendrológicos vegetativos de Jacaranda mimosifolia: A-hoja; B-foliolos; C-raquis y D-corteza.

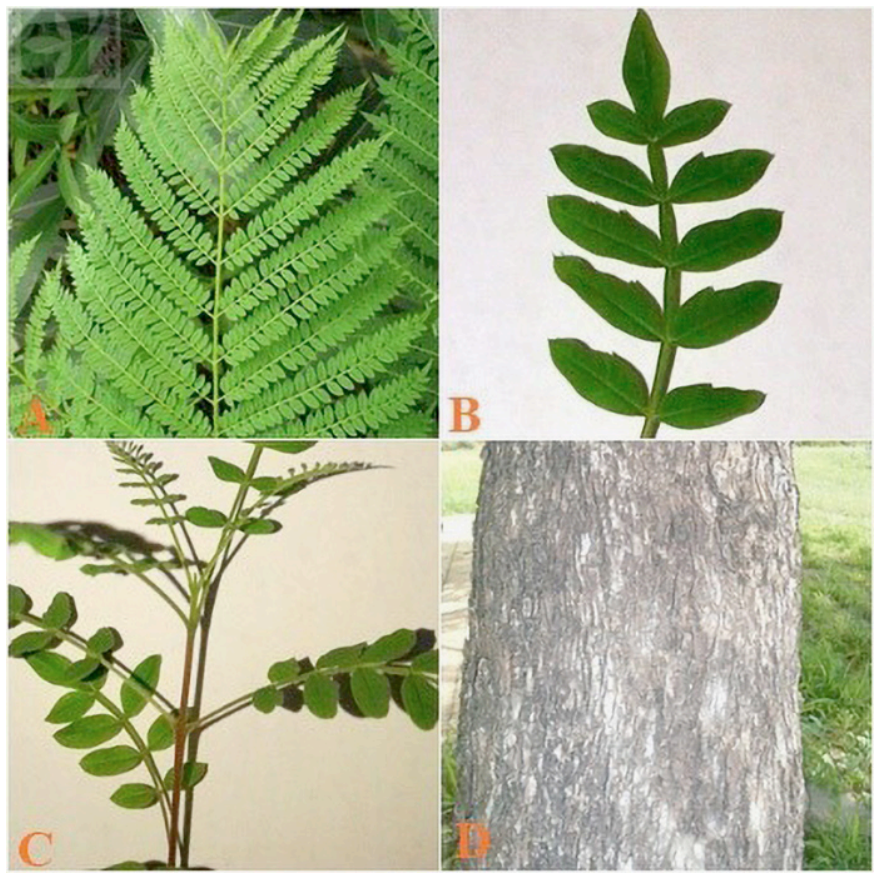


Figura 6. Detalle de caracteres dendrológicos reproductivos de Peltophorum dubium:

A-inflorescencia; B-flor; C-fruto y D-semilla.

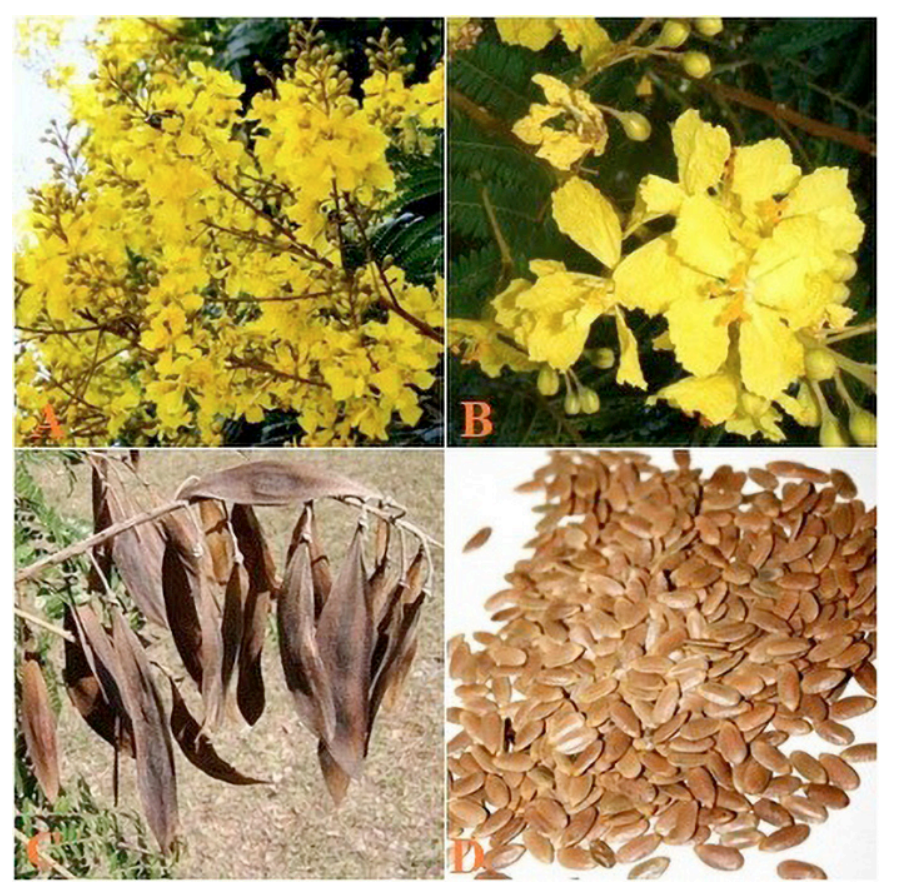

Figura 8. Detalle de caracteres dendrológicos reproductivos de Enterolobium contortisiliquum: A-inflorescencia; B-flor; C-fruto y D-semilla.

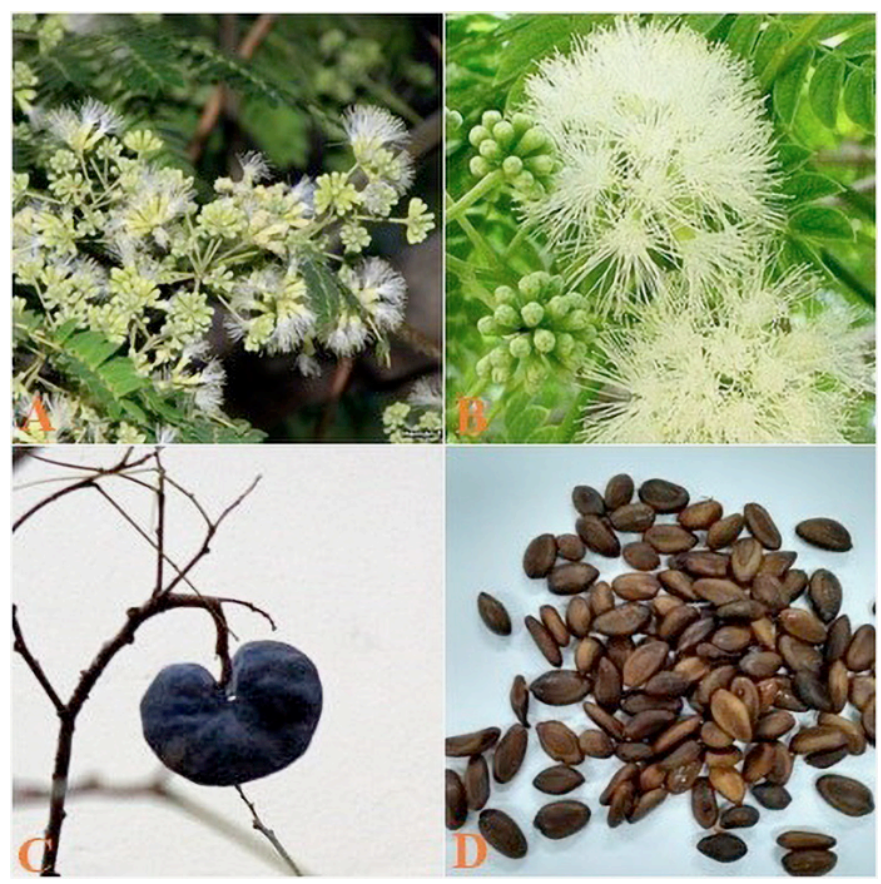

Figura 7. Detalle de caracteres dendrológicos reproductivos de Delonix regia: A-inflorescencia; B-flor; C-fruto y D-semilla.

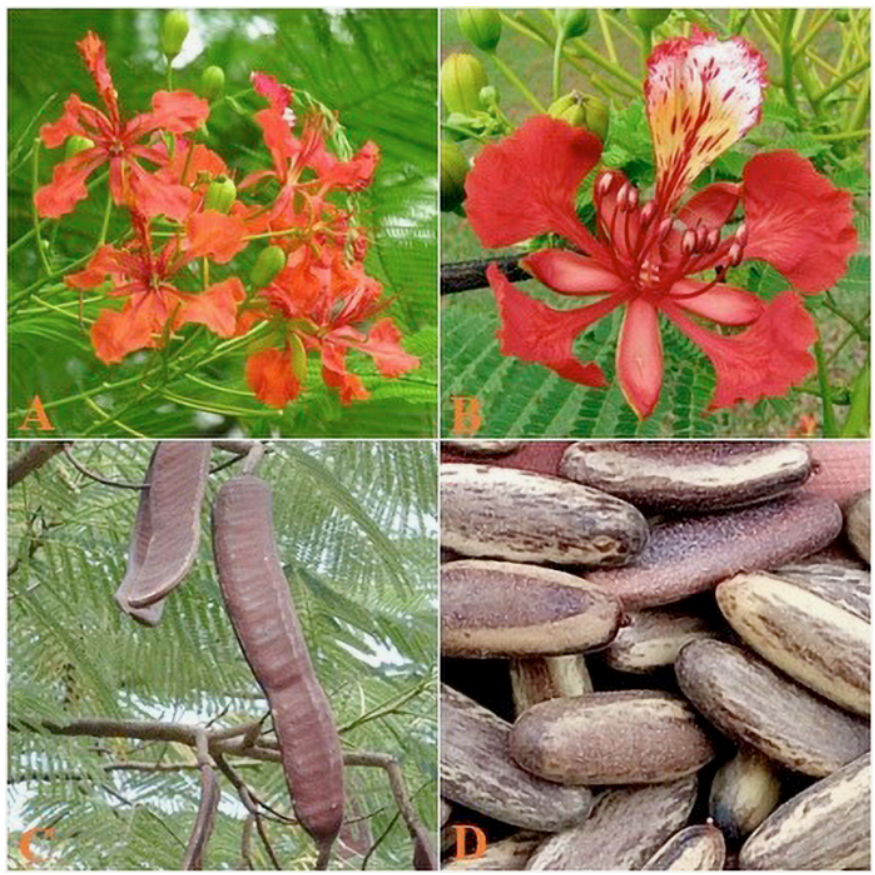

Figura 9. Detalle de caracteres dendrológicos reproductivos de J. mimosifolia: A-inflorescencia; B- flor; C-fruto y D-semilla.

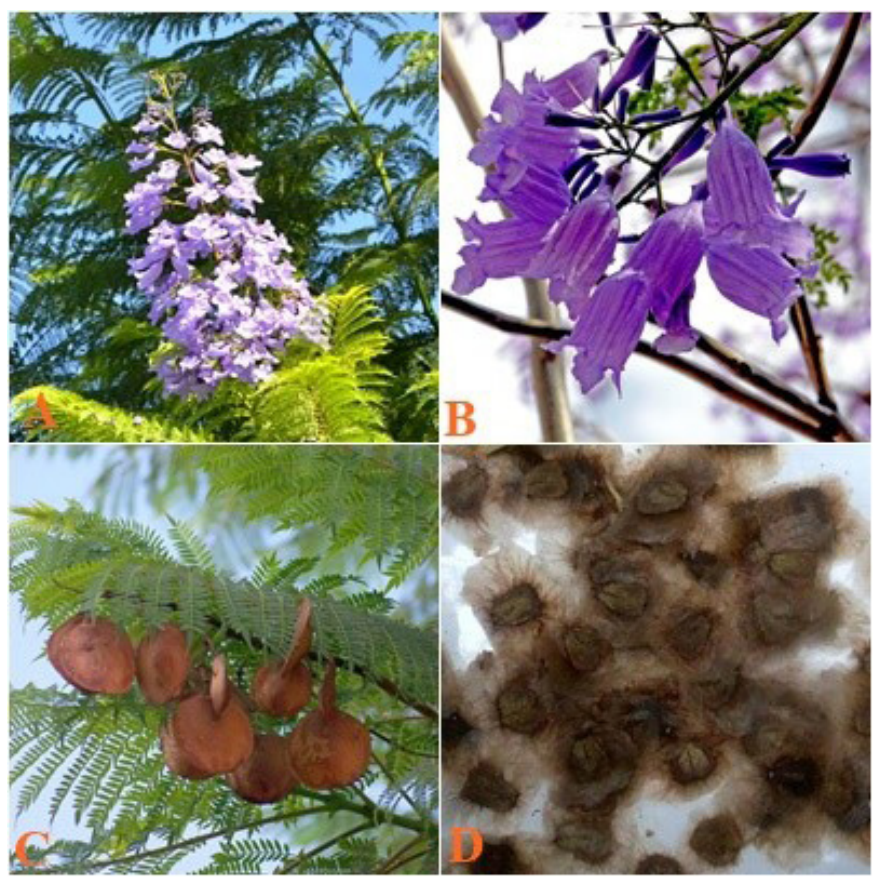


Clave dendrológica con caracteres vegetativos para el Arboretum FCA-UNNE.

A-Hojas alternas.

B-Foliolos con nervadura céntrica, corteza parda

(Figuras 2 D).

C-Foliolos/foliólulos asimétricos (1ํㅜ par de foliólulos más próximos al raquis asimétrico)

(Figuras 2 A, B).

D-Raquis piloso (Figura $2 \mathrm{C}$ ).

\section{Peltophorum dubium}

CC - Foliolos/foliólulos simétricos, corteza parda

(Figuras 3 A, B, D).

DD-Raquis glabro (Figura $3 \mathrm{C}$ ).

Delonix regia

BB-Foliolos con nervadura excéntrica, corteza gris clara a pardo grisácea (Figuras 4 A, B, C, D).

Enterolobium contortisiliquum

AA-Hojas opuestas, raquis glabro, corteza grisácea (Figuras 5 A, B, C, D).

Jacaranda mimosifolia

\section{Clave dendrológica con caracteres} reproductivos para el Arboretum FCA-UNNE.

A- Inflorescencias simples.

B-Flores en racimos terminales, axilares o ambos

(Figura 7 A).

C-Flores rojas (Figura 7 B).

D-Frutos vainas dehiscentes (Figura 7 C, D). Delonix regia

BB - Flores en capítulo terminal (Figura 8 A).

CC-Flores blancas (Figura 8 B).

DD- Frutos vainas indehiscentes (Figuras 8 C, D).

Enterolobium contortisiliquum

AA- Inflorescencias compuestas.

B- Flores en panículas terminales (Figura 6 A).

C- Flores amarillas.

DD- Frutos cápsulas indehiscentes, semillas sin alas (Figuras 6 B, C, D).

Peltophorum dubium

BB- Flores en panículas axilares (Figura 9 A).

CC- Flores azules.
DD- Frutos cápsulas dehiscentes, semillas con alas (Figuras 9 B, C, D).

\section{Jacaranda mimosifolia}

\section{CONCLUSIÓN}

La confección de las claves de identificación tanto con caracteres vegetativos y reproductivos permite su uso práctico para la identificación de las especies cuatro especies arbóreas presentes en el Arboretum, independiente del estadio fenológico en que pudieran encontrarse.

\section{REFERENCIAS BIBLIOGRÁFICAS}

Acosta-Hernández, C. 2013. Especies no aptas y con manejo especial para la arborización urbana de Montería, Colombia. Revista nodo, 15 (8-8): 65-76

Aguirre, Z. 2012. Especies forestales de los bosques secos del Ecuador. Guía dendrológica para su identificación y caracterización. Finlandia. Quito, Ecuador: Proyecto Manejo Forestal Sostenible ante el Cambio Climático. MAE/ FA0. 140pp.

Amurrio Mamani, D. 2009. Componentes de la vegetación arborea, arbustiva y de regeneración natural en sistemas agroforestales sucesionales en la comunidad de Combuyo - Cochabamba. Documento elaborado para obtenerel Titulo de Técnico Superior Forestal. Universidad Mayor de San Simón, Escuela de Ciencias Forestales, Cochabamba, Bolivia. 59pp.

APN-SIB (Administración de Parques Nacionales. Sistema de Información de Biodiversidad). 2016. Listado de especies registradas. (https:// sib.gob.ar/especies). Acceso: 08/08/2019.

Asesor, P. 2019. Tarco, jacarandá-Jacaranda mimosifolia. Universo Tucumano $\mathrm{N}^{\circ} 22.10 \mathrm{pp}$. 
Asociación Española de Arboricultura. 2014. Jacaranda Mimosifolia: Características y utilización. Ficha tecnica. 2 p.

Baluarte Vásquez, J. 1993. Características dendrologicas de cinco especies forestales de selva baja. Folia Amazonica, 5 (1-2), 59-74. DOI: https://doi.org/10.24841/fa.v5i1-2.221

Cabrera, M.; Andrada, A.; Gallez, L. 2013. Floración de especies con potencial apícola en el Bosque nativo formoseño, distrito chaqueño oriental (ArgentIna). Boletin de la Sociedad Argentina de Botánica 48 (3-4): 477-491. DOI: https:// doi.org/10.31055/1851.2372.v48.n3-4.7554

Cálix Pizatti, R. 1970. Identificación dendrológica y anatómica de 37 especies arbóreas de Honduras. Tesis Magíster Agrícola. Instituto Interamericano de Ciencias Agrícolas. 180pp.

Carvalho, R. 1994. Especies forestáis brasileiras. Recomendacões silvicultirais, potencialidades $e$ uso de madeira. Brasil. EMBRAPA 639pp.

Comellas García-Llera, J. 2011. Historia de los cambios climáticos. Ediciones Rialp. 320pp.

Damião Filho, C. F.; Môro, F. V. 2005. Morfologia vegetal. Jaboticabal: Funep, 172 p.

Dueñas Linares, H.; Nieto Ramos, C. 2010. Dendrología tropical: "estudio y caracterización dendrológica de las principales especies forestales de la amazonía peruana". (H. H. Linares, Ed.) Puerto Maldonado, Perú. 244pp.

Duke, J. 1969. On tropical tree seedling. Seeds, seedlings, systems and systematic. Annals of the Missouri Botanical Garden, 55: 125-65.

Duke,J.; Polhill,R. 1981.SeedlingsofLeguminoseae. Advances in Legume Systematics. Ed. R.M. Polhill \& P.H. Raven. Pp 941-949.

Egg, W. 1994. Experiencias agroforestales exitosas en la cuenca amazónica. Tratado de Cooperacion Amazonica. Secretaria ProTempore № 23. 195pp.

Elbert, L.; Little, Jr.; Franic, H.; Marrero, J. 1977. Árboles comunes de Puerto Rico y las Islas Vírgenes. Servicio Forestal Departamento de Agricultura de los Estados Unidos de Norteamérica. Delonix regia. 215pp.

EOL (Encyclopedia of Life), 2020. Delonix regia (Bojer ex Hook.) Raf. (https:// stricollections.org/portal/taxa/index. php?taxauthid=1\&taxon=63921\&clid=71). Acceso: 21/01/2020

Escobar, E.; Ligier, D.; Melgar, R.; Matteio, H.; Vallejos, 0. 1996. Mapa de suelos de la Provincia de Corrientes 1:500000. Corrientes: INTACorrientes. (http://www.geointa.inta.gob. ar/2016/09/23/suelos-de-la-provincia-decorrientes-1500-000/). Acceso: 19/10/2019.

Fontúrbel, F.E.; Achá, D.; Mondaca, D.A. 2007. Manual de Introducción a la Botánica. La Paz, Bolivia. Publicaciones Integrales. 252pp.

Gartland, H. 2008a. Dendrología general: nomenclatura especial de los árboles forestales - 1a ed. - Posadas: EDUNaM - Editorial Universitaria de la Universidad Nacional de Misiones, 120pp.

Gartland, H. 2008b. Dendrología especial: especies forestales de Argentina - 1a ed. Posadas: EdUNaM - Editorial Universitaria de la Universidad Nacional de Misiones,132 p.

Giménez, A.; Moglia, J. 2003. Árboles del Chaco Argentino: Guía para el reconocimiento dendrológico. Santiago del Estero - Argentina: Universidad Nacional de Santiago del Estero. 316pp.

Grau, A.; Kortsarz, A.M. 2012. Capítulo 8: Las especies de árboles de Tucumán. In: Guía de arbolado de Tucumán. Grau A.; Kortsarz A.M. (Eds.). Universidad Nacional de Tucumán, Argentina. p. 108-229.

Hoyos, J. 1979. Arboles de Caracas. Delonix regia. $134 \mathrm{pp}$.

IUCN, 2017. The IUCN Red List of Threatened Species. Version 2017-3. (www.iucnredlist. org). Acceso: 08/03/2019.

Juárez de Varela, F. 2012. Bignoniaceae Juss. Aportes Botánicos de Salta. Serie Flora. Vol. 2 
$\mathrm{N}^{\circ} 22$. Edición digital. Herbario MCNS, Facultad de Ciencias Naturales, Universidad Nacional de Salta. Salta, Argentina. 40pp.

Killeen, T.; García, E.; Beck, S. 1993. Guía de árboles de Bolivia. Ed: Herbario Nacional de BoliviaMissouri Botanical Garden. 958pp.

Kostlin, M. 2017. IdentIfIcaclón de LegumInosaecaesalpinioideae arbustlvas-arbóreas y LIanas de La selva paranaense (Misiones, Argentina) utilizando caracteres vegetativos. Bol. Soc. Argent. Bot. 52 (3): 597-615.

Kroll, B.; Marrinillod, D.; Nalvarte, W. 1992. Apuntes dendrológicos del Perú; nombres vernaculares y especies de Dantas, Lima. Perú. Lima, Perú. 246pp.

Lema Aucacama, A. 2013. Separación y posible identificación de metabolitos secundarios de la Jacaranda (Jacaranda mimosifolia) con fines de aporte a una técnica de análisis químico. Tesis Pregrado, Escuela Superior Politécnica de Chimborazo. Facultad de Ciencias. Escuela de Bioquímica y Farmacia. Riobamba, Ecuador. 120pp.

López, J.; Little Jr. E.; Ritz, G.; Rombold, J.; Hahn, W. 1987. Árboles comunes del Parguay. Ñande Yvyra Mata Kuera. Cuerpo de Paz. Paraguay. 425 pp.

Lozano, E.; Zapater, M.; Mamani, C.; Flores, C.; Gil, M.; Sühring, S. 2016. Efecto de pretratamientos en semillas de Enterolobium contortisiliquum (Fabaceae) de la selva pedemontana Argentina. Boletín de la Sociedad Argentina de Botánica, 51(1):79-87.

Marzocca, A. 1985. Nociones Básicas de Taxonomía Vegetal. San José, Costa Rica. IICA. 263p.

Mostacedo, B.; Justianiano, J.; Toledo, M.; Fredericksen, T. 2003. Guía Dendrólogica de Especies Forestales de Bolivia (2da. Edición). Santa Cruz, Bolivia. 204pp.

Murphy, G. 2008. Atlas Agroclimático de la Argentina. Editorial Facultad de AgronomíaUBA. 250pp.
Nultsch, W. 2000. Botânica Geral. Porto Alegre: Artmed. 10 ed., 489 p.

Parra, P. 1983. Estudio de la morfologia externa de plantulas de Calliandra gracilis, Mimosa albida, Mimosa arenosa, Mimosa camporum y Mimosa tenuiflora. Morfologia Vegetal de la Facultad de Agronomia, UCV, Maracav. Venezuela.

Peña, J. 2013. La importancia de la dendrología en la carrera profesional del ingeniero forestal. Facultad de Ciencias Forestales de la Universidad Nacional Agraria, La Molina, Perú.

Piedrahita, E.; Soto Garcia, E. 1987. Estudios fisiologicos y anatomicos de las semillas $y$ plantulas de Roble Tabebuia rosae Bertol y Cedro Cedrela odorata L. Medellin. Colombia. Facultad de Agronomia. Pp 110.

Poulsen, K.; Parratt, M.; Gosling, P. 1998. ISTA Tropical and subtropical tree and shrub handbook. Zürich, Suiza. 203pp.

Prado, D. 1998. Jacaranda mimosifolia. The IUCN Red List of Threatened Species. e. T32027A9675619. (https://dx.doi.org/ 10. 2305/IUCN.UK.1998.RLTS.T32027A9675619. en). Acceso: 08/08/2019.

Quesada Monge, R.; Fernández Vega, J. 2005. Actualización de listado de especies arbóreas de uso forestal y otros usos en Costa Rica. Kurú: Revista Forestal, 2(5): 44.

Ríos, J. 1990. Prácticas de Dendrología tropical. Cooperación técnica suiza - REDINFOR. UNA. La Molina. Lima, Perú. 145. p.

Rivers, M. 2014. Delonix regia. The IUCN Red List of Threatened Species 2014: e. T32947A2828337. (https://dx.doi.org/10. 2305/IUCN.UK.2014-1.RLTS.T32947A2828 337.en). Acceso: 08/08/2019.

Rodríguez Aranguren, S.; Gámez Álvarez, L. 2010. Clave vegetativa para la identificación de árboles de la familia Fabaceae de la ciudad de Mérida, Venezuela. Pittieria, 34: 89-111.

Roskov, Y.; Abucay, L.; Orrell, T.; Nicolson, D.; Flann, C.; Bailly, N.; Kirk, P.; Bourgoin, T.; De 
Walt, R.; Decock, W.; De Wever, A. 2016. Species 2000 \& ITIS Catalogue of Life, 28th July 2016. (http://www.catalogueoflife.org/col). Acceso: 08/08/2019.

Salazar, R.; Soihet, C. 2001. Manejo de semillas de 75 especies forestales de América Latina. Volumen II. Turrialba, Costa Rica: CATIE. Proyecto de Semillas forestales: Danida Forest Seed Centre. 155pp.

Sauñe Ferrel, A. 2013. Caracterización Dendrológica y claves de identificación de las especies del género Piper en los Valles de Chanchamayo y Satipo - Junín. Tesis Pregrado. Universidad Nacional Agraria La Molina. Facultad de Ciencias Forestales. Lima, Perú. 197pp.

Silva Costa, R.; Ortolani, F.A.; Vitti Môro, F.; de Paula, R.C. 2011. Caracterização morfológica de folhas e flores de espécies de Jacaranda (Bignoniaceae), cultivadas em Jaboticabal - SP. Revista de Biologia e Ciências da Terra, 11(1):169-181.

SINAC (Sistema Nacional de Áreas de Conservación y Programa REDD-CCAD-GIZ). 2014. Protocolo de campo para la identificación de especies arbóreas: Información taxonómica y dendrológica de las especies arbóreas de Costa Rica. San José, Costa Rica.

Sugita, N.; Burgos, A.; Luna, C. 2018. Diseño y ejecución del Arboretum de la Facultad de
Ciencias Agrarias de la Universidad Nacional del Nordeste, Corrientes, Argentina. Revista de Agronomía del Noroeste Argentino, 38(1): 2332.

Tapia, W.; Armas, G. 2014. Estudio de la actividad antibacteriana y tóxica del Kuiship Uacaranda copaia). La Granja: Revista de Ciencias de la Vida 19(1): 12-20.

UICN, 2016. IUCN Red List of Threatened Species. (http://www.iucnredlist.org/search). Acceso: 08/08/2019.

Ventoso, A.; Mongiardino, C. 2014. Guia de identificación de especies arboreas nativas: Uruguay. MVOTMA. DINAMA, Montevideo. Tradinco. 166pp.

Yajure, Y.; Gámez, L. 2011. Determinación de las Bignoniaceae de la ciudad de Mérida (Venezuela) por medio de caracteres vegetativos. Pittieria, 35: 13-24.

Yépez, P. 2011. Las plantas en las creencias y mitos del Ecuador. (http://www.biologia.puce.edu. ec/natura.php?c=350i). Acceso: 08/08/2019.

Zarate Gómez, R.; Mori Vargas, T.; Ramírez Arevalo, F.; Davila Doza, H.; Gallardo Gonzales, G.; Cohello Huaymacari, G. 2015. Lista actualizada y clave para la identificación de 219 especies arbóreas de los bosques sobre arena blanca de la Reserva Nacional Allpahuayo Mishana, Loreto, Perú. Acta Amazonica 45(2): 133-156. DOI: https:// doi.org/10.1590/1809-4392201402922

Recibido: 27 de septiembe de 2019 Aceptado para publicación: 14 de febrero de 2020

Esta obra está bajo una Licencia Creative Commons Atribución-NoComercial-SinDerivar 4.0 Internacional. 